\title{
A STRONG CONTRACTIVITY PROPERTY FOR SEMIGROUPS GENERATED BY DIFFERENTIAL OPERATORS
}

\author{
ROBERT M. KAUFFMAN
}

\begin{abstract}
Frequently, nonconservative semigroups generated by partial differential operators in $L_{2, \rho}\left(R^{k}\right)$ have the property that initial conditions which are large at $|x|=\infty$ become immediately small at infinity for all $t>0$. This property is related to the rate of decay of eigenfunctions of the differential operator. In this paper this phenomenon is investigated for a large class of differential operators of second and higher order. New estimates on the rate of decay of the eigenfunctions are included, which are related in special cases to those of Agmon.
\end{abstract}

0. Introduction. Frequently, in nonconservative abstract Cauchy problems, singular behavior is suppressed. We deal with a phenomenon in this paper which is best described as the suppression of singularities at infinity.

Suppose that the abstract Cauchy problem is formulated in $L_{2, \rho}\left(R^{k}\right)$, where $\rho$ is a bounded positive measurable function such that $\rho(x)$ approaches zero as $|x|$ approaches infinity. The phenomenon we study here is that when $u(0) \in L_{2, \rho}\left(R^{k}\right)$, with norm $\|f\|=\left(\int_{R^{k}}|f(x)|^{2} \rho(x) d x\right)^{1 / 2}, u(t) \in L_{2,1 / \rho}\left(R^{k}\right)$ for all $t>0$. Hence, while $u(0)$ may be allowed to grow rapidly at infinity (depending on $\rho), u(t)$ must decay rapidly at infinity for all $t>0$.

That this behavior occurs at all is rather surprising. In this paper, it will be shown to occur frequently when the infinitesimal generator is an ordinary or partial differential operator with positive coefficients, in the discrete spectrum case.

Although we study this phenomenon as a mathematical one, it is also related to such areas as heat conduction and nonconservative quantum mechanics. For example, in heat conduction it deals with the case where an initial temperature which is initially large at infinity becomes immediately small at infinity. The physical interpretation for this is that the equation represents the temperature of a twodimensional thin slab with variable thermal conductivity, surrounded by a medium at constant temperature with variable rates of conduction via Newton's law of cooling to the surrounding medium. Under hypotheses of the theorems, this conduction approaches perfect conduction at $|x|=\infty$; hence this instantaneous decrease in temperature might be expected. Nevertheless, it seems reasonable that there is an initial growth rate at infinity, determined by $\rho$, beyond which we would not expect the phenomenon to occur. A good deal of the paper is involved with the computation of $\rho$ from the coefficients of the infinitesimal generator. $\rho$ turns out to decay very rapidly at infinity.

Received by the editors July 16, 1986 and, in revised form, March 11, 1987. Presented to the International Conference on Differential Equations and Mathematical Physics, Birmingham, Alabama, March 3-8, 1986, sponsored by the NSF, UAB and UA.

1980 Mathematics Subject Classification (1985 Revision). Primary 47F05, 47D05, 35P20, 35K30. 
We wish to show that this phenomenon occurs not just in heat conduction problems, but is a mathematical phenomenon which occurs quite widely. In fact, we shall show that it occurs in abstract Cauchy problems $u^{\prime}(t)=-\lambda L u(t), u(0)=f$, in $L_{2, \rho}\left(R^{k}\right)$, where $\lambda$ has positive real part and $L$ is an arbitrary order, positive coefficient differential operator where the potential or 0 th order term is larger in a certain sense than the higher order terms, and where $\rho$ is computed fairly easily from the coefficients of $L$.

The mathematical cause of this phenomenon is the extremely rapid decay at infinity of the eigenfunctions of $L$. This decay, which is new even in the second-order case, is established in $\S 5$ of this paper, in a relatively self-contained manner. The method of proof is new and functional-analytic; it depends strongly upon the essential selfadjointness in $L_{2}\left(R^{k}\right)$ of the restriction of $L$ to $C_{0}^{\infty}\left(R^{k}\right)$, a fact which is also established here. While the paper is relatively self-contained from the standpoint of differential equations, basic material from semigroup theory concerning $m$-sectorial operators and the abstract Cauchy problem will be used freely. Goldstein [5] and Kato [7] are good references for this material.

In attempting to study the nature of the phenomenon, it is perhaps instructive to consider one more elementary application, this time loosely related to quantum mechanics. It is possible to interpret the equation $u^{\prime}=-\lambda L u, u(0)=f$, with $\operatorname{Re}(\lambda)=\varepsilon>0$, as a slightly nonconservative Schroedinger equation, where the rate of loss of probability in a pure state $\psi_{n}$ is proportional to the product of the eigenvalue $\lambda_{n}$ associated with $\psi_{n}$ and total probability $\left\|\psi_{n}\right\|^{2}$ of $\psi_{n}$. The physical interpretation here is that even a badly nonnormalizable initial state $f$ immediately becomes highly localized, because the high-energy states tend to disappear and the low-energy states are trapped in a potential well. The nonnormalizability disappears because it is associated with the high-energy states. These states are themselves in $L_{2,1 / \rho}\left(R^{k}\right)$, but may have a large norm in this space.

Although the specific phenomenon of this paper may not have been studied before, at least in any generality, it is somewhat related to the general study of various types of contractivity which was initiated by E. Nelson (see [9]), and which has been studied by a number of authors, most recently Davies and Simon [3]. The estimates on the rate of decay (and growth) of the eigenfunctions, which form the mathematical heart of the paper, are due to S. Agmon (see [1 and 2]) in the uniformly elliptic second order PDE case. They seem new in the higher order ODE and PDE cases, and in the second order nonuniformly elliptic case.

The method of proof of the decay of the eigenfunctions rests upon the fact that the restriction of $L$ to $C_{0}^{\infty}\left(R^{k}\right)$ is essentially selfadjoint. Later, to gain some estimates on the spectrum of $L$, we shall need this assertion for all powers of $L$. The assertion for $L$ is new in the higher order ODE and PDE cases, though it is very similar to a special case of a general higher order result of the author in [8]; in this general result the potential term need not be dominant. Evans [4], Kato [6] and Read [10] have all proved second-order results of the same general type as the one of this paper, which seem to apply to somewhat different classes of expressions. The methods of proof of essential selfadjointness used here are those of the author in [8].

The author wishes to thank W. D. Evans for several interesting discussions about this paper, and Mark Kon for pointing out the work of Davies and Simon mentioned above. 
1. A theorem about semigroups. In this section we state and prove a theorem about semigroups generated by differential operators, which gives the "suppression of singularities at infinity" discussed in the introduction. The remainder of the paper will be devoted to showing that a large class of differential expressions $L$ satisfy the hypotheses of this theorem, and to showing how to calculate $\rho$ from the coefficients of $L$.

NOTATION 1.1. Throughout the paper, let $\omega$ be a positive $C^{\infty}$ function with domain $R^{k}$, such that $\omega(x)$ approaches infinity as $|x|$ approaches infinity. Let $\rho=\omega^{-2}$. Throughout $\S 1$, let $L$ denote a differential expression $L=\sum_{|\alpha| \leq N} a_{\alpha} D^{\alpha}$, where $a_{\alpha} \in C^{\infty}\left(R^{k}\right)$ for any $\alpha=\langle\alpha(1), \ldots, \alpha(k)\rangle$, where each $\alpha(i)$ is a nonnegative integer, and $D^{\alpha}=D_{1}^{\alpha(1)} \cdots D_{k}^{\alpha(k)}$, with $D_{i}=\partial / \partial x_{i}$. In $\S 1$, we suppose that the restriction of $L$ to $C_{0}^{\infty}\left(R^{k}\right)$ is essentially selfadjoint and bounded below in $L_{2}\left(R^{k}\right)$; let $H$ denote the closure of this restriction.

DEFINITION 1.2. Let $\lambda$ be a complex number with $\operatorname{Re} \lambda>0$, and use the notation of Notation 1.1. The $d u(t) / d t=-\lambda L u(t), u(0)=f$, in $L_{2, \rho}\left(R^{k}\right)$, is said to be well posed if

(i) for every $f$ in $L_{2, \rho}\left(R^{k}\right)$ there exists a unique function $u$ with domain $[0, \infty)$ and range contained in $L_{2, \rho}\left(R^{k}\right)$ such that

(ia) $u(0)=f$

(ib) $u$ is continuous;

(ic) $u$ is differentiable at all $t>0$;

(id) $L u(t) \in L_{2, \rho}\left(R^{k}\right)$ for all $t>0$;

(ie) $u^{\prime}(t)=-\lambda L u(t)$ at all $t>0$;

(ii) if $v(t) f$ is defined to be that $u(t)$ such that $u(0)=f$, for $t>0$, then for any $t>0 v(t)$ is a bounded linear transformation with domain $L_{2, \rho}\left(R^{k}\right)$ and range contained in $L_{2, \rho}\left(R^{k}\right)$.

DEFINITION 1.3. Let $\lambda$ be a complex number with positive real part, and suppose that the abstract Cauchy problem $d u(t) / d t=-\lambda L u(t), u(0)=f$, in $L_{2, \rho}\left(R^{k}\right)$ is well posed. The problem will be said to be $\rho$-contractive if $u(t) \in$ $L_{2,1 / \rho}\left(R^{k}\right)$ for all $f \in L_{2, \rho}\left(R^{k}\right)$ and all $t>0$.

THEOREM 1.4. Suppose that L, H, $\omega$ and $\rho$ are as in Notation 1.1. Suppose in addition that (i)-(iv) hold:

(i) the closure $A$ of the restriction of $\omega L \omega^{-1}$ to $C_{0}^{\infty}\left(R^{k}\right)$ is an m-sectorial operator in $L_{2, \rho}\left(R^{k}\right)$;

(ii) $(H+r I)^{-1}$ is compact in $L_{2}\left(R^{k}\right)$ for some $r>0$ (hence in particular $H$ has an orthonormal basis of eigenvectors);

(iii) every eigenfunction of $H$ is in $L_{2,1 / \rho}\left(R^{k}\right)$;

(iv) if $\|f\|_{1 / \rho}$ refers to the norm of $f$ in $L_{2,1 / \rho}\left(R^{k}\right)$, and if $\left\{\lambda_{n}\right\}_{1}^{\infty}$ is the spectrum of $H$, listed according to multiplicity in nondecreasing order (so that if $\lambda$ has multiplicity $s, \lambda$ is listed s times), and $\left\{f_{n}\right\}$ is an orthonormal basis for $L_{2}\left(R^{k}\right)$ such that $f_{n}$ is an eigenvector of $H$ with eigenvalue $\lambda_{n}$, then $\sum_{n=1}^{\infty} \exp \left(-\varepsilon \lambda_{n}\right)\left\|f_{n}\right\|_{1 / \rho}<\infty$ for all $\varepsilon>0$.

Then, for any $\lambda$ such that $\operatorname{Re} \lambda>0$, the abstract Cauchy problem $d u(t) / d t=$ $-\lambda L u(t), u(0)=f$, is well posed and $\rho$-contractive in $L_{2, \rho}\left(R^{k}\right)$. 
PROOF. Let $T_{0}\left(\omega L \omega^{-1}\right)$ denote the closure of the restriction of $\omega L \omega^{-1}$ to $C_{0}^{\infty}\left(R^{k}\right)$

We define $T_{1}\left(\omega^{-1} L \omega\right)$ to be the restriction of $\omega^{-1} L \omega$ to $\left\{f \in L_{2}\left(R^{k}\right) \mid \omega^{-1} L \omega f \in\right.$ $\left.L_{2}\left(R^{k}\right)\right\}$, where the derivatives are taken in the distribution sense. Define $T_{1, \rho}(L)$ to be the restriction of $L$ to $\left\{f \in L_{2, \rho}\left(R^{k}\right) \mid L f \in L_{2, \rho}\left(R^{k}\right)\right\}$, where the derivatives are again taken in the distribution sense.

We show that $T_{1, \rho}(L)$ is $m$-sectorial. If $f$ is in the domain of $T_{1, \rho}(L)$, then $\omega^{-1} f \in L_{2}$ and $L f=L \omega\left(\omega^{-1} f\right) \in L_{2, \rho}$. Hence $\omega^{-1} L \omega\left(\omega^{-1} f\right) \in L_{2}$. Thus $T_{1, \rho}(L) \subseteq \omega\left(T_{1}\left(\omega^{-1} L \omega\right)\right) \omega^{-1}$. The opposite inclusion is obvious; hence $T_{1, \rho}(L)$ is unitarily equivalent to $T_{1}\left(\omega^{-1} L \omega\right)$. But $T_{1}\left(\omega^{-1} L \omega\right)$ is $T_{0}\left(\omega L \omega^{-1}\right)^{*}$. In fact, it is clear that $T_{1}\left(\omega^{-1} L \omega\right) \subseteq T_{0}\left(\omega L \omega^{-1}\right)^{*}$. But by the definition of the distributional derivative, since the coefficients of $\omega L \omega^{-1}$ and $\omega^{-1} L \omega$ are in $C^{\infty}$, it follows that $T_{0}\left(\omega L \omega^{-1}\right)^{*} \subseteq T_{1}\left(\omega^{-1} L \omega\right)$. By Theorem 2.5, p. 323, Kato [7], the adjoint of an $m$-sectorial operator is also $m$-sectorial. Hence $T_{1}\left(\omega^{-1} L \omega\right)$ and $T_{1, \rho}(L)$ are $m$ sectorial.

But any $m$-sectorial operator in a Hilbert space is the infinitesimal generator of a holomorphic semigroup. Also, the abstract Cauchy problem stated in Definition 1.2 is well posed if $T_{1, \rho}(L)$ is the generator of a holomorphic semigroup. We need only show that $v(t) f \in L_{2,1 / \rho}\left(R^{k}\right)$ for all $t>0$, where $v(t)$ is defined in Definition 1.2 .

If $f \in L_{2}\left(R^{k}\right), v(t) f=\sum_{n=1}^{\infty} c_{n} \exp \left(-\lambda \lambda_{n} t\right) f_{n}$, where $c_{n}=\left(f, f_{n}\right)$. But if $f \in L_{2, \rho}\left(R^{k}\right), f \bar{f}_{n} \in L_{1}\left(R^{k}\right)$ and $\left|\int f \bar{f}_{n}\right| \leq\left\|f_{n}\right\|_{1 / \rho}\|f\|_{\rho}$. Hence, for any $t>0$ and $f \in L_{2, \rho}\left(R^{k}\right)$,

$$
\sum_{1}^{\infty}\left|\int f \bar{f}_{n}\right|\left|\exp \left(-\lambda \lambda_{n} t\right)\right|\|f\|_{1 / \rho} \leq\|f\|_{\rho} \sum_{1}^{\infty}\left\|f_{n}\right\|_{1 / \rho}^{2} \exp \left(-\varepsilon \lambda_{n} t\right) \leq K\|f\|_{\rho}
$$

where $\varepsilon=\operatorname{Re} \lambda$. Hence the mapping $T$ taking $f$ to $\sum_{1}^{\infty}\left(\int f \bar{f}_{n}\right) \exp \left(-\lambda \lambda_{n} t\right) f_{n}$ is a bounded linear transformation taking $L_{2, \rho}\left(R^{k}\right)$ into $L_{2,1 / \rho}\left(R^{k}\right)$. But, since $v(t)$ is bounded, and $L_{2}\left(R^{k}\right)$ is dense in $L_{2, \rho}\left(R^{k}\right)$, we see that $v(t) f=T(f)$ for all $f$ in $L_{2, \rho}\left(R^{k}\right)$. Thus $v(t) f \in L_{2,1 / \rho}\left(R^{k}\right)$ for all $t>0$. The theorem is proved.

2. Statement of main theorems. In this section we give a large class of differential expressions $L$ which satisfy the hypotheses of Theorem 1.4, and show how to calculate $\omega$ from the coefficients of $L$. In this section we state the main theorems about $L$, including those concerning $\rho$-contractivity and growth and decay of the eigenfunctions, numbering the theorems according to the later section where they are proved.

DEFinition 2.1. Throughout the rest of the paper, $L$ will denote an ordinary or partial differential expression of the form

$$
L=\sum_{i=1}^{k} \sum_{j=1}^{N(i)}(-1)^{j} D_{i}^{j} p_{i j}^{2} D_{i}^{j}+p_{0}^{2}
$$

where $D_{i}=\partial / \partial x_{i}$ and $p_{i j}$ and $p_{0}$ are functions in $C^{\infty}\left(R^{k}\right)$, which are either positive or identically zero, with $p_{i N(i)}$ positive and $N(i) \geq 1$ for each $i$. We assume the following:

(i) $p_{0}(x)$ approaches infinity as $|x|$ approaches infinity; 
(ii) $N(i) \geq 1$ for all $i \in 1, \ldots, k$;

(iii) for each $i \in 1, \ldots, k$, there exists a function $\varphi_{i}^{*}$ in $C^{\infty}\left(R^{1}\right)$ such that (iiia) $\varphi_{i}^{*}(x)>0$ for all $x$;

(iiib) $\left(\varphi_{i}^{*}\right)^{(j)}=O\left(\left(\varphi_{i}^{*}\right)^{j+1}\right)$ for all $j \geq 1$, where $\varphi_{i}^{*(j)}$ denotes the $j$ th derivative of $\varphi_{i}^{*}$ and $\left(\varphi_{i}^{*}\right)^{j+1}$ denotes the $(j+1)$ st power;

(iiic) for any multi-index $\xi, D^{\xi} p_{i j}=O\left(\varphi^{\xi} p_{i j}\right)$ for all $i \in 1, \ldots, k$ and $j \in$ $1, \ldots, N(i)$; also $D^{\xi} p_{0}=O\left(\varphi^{\xi} p_{0}\right)$, where $\varphi^{\xi}$ is defined by $\varphi^{\xi}=\prod_{i=1}^{k} \varphi_{i}^{\xi(i)}$, and $\varphi_{i} \in C^{\infty}\left(R^{k}\right)$ is defined by $\varphi_{i}(x)=\varphi_{i}^{*}\left(x_{i}\right)$;

(iv) $p_{i j} \varphi_{i}^{j}=o\left(p_{0}\right)$ for all $j \geq 1$.

DEFINITION 2.2. Define $\alpha_{i}^{*}(x) \in C^{\infty}\left(R^{1}\right)$ by the following:

(i) $\alpha_{i}^{*}(x)=\int_{0}^{x} \varphi_{i}^{*}(s) d s$ for $x \geq 1$;

(ii) $\alpha_{i}^{*}(x)=\int_{x}^{0} \varphi_{i}^{*}(s) d s$ for $x \leq-1$;

(iii) $\alpha_{i}^{*}(x) \in C^{\infty}\left(R^{1}\right)$;

(iv) $\alpha_{i}^{*}(x)>0$ for all $x$.

Define $\alpha_{i}(x) \in C^{\infty}\left(R^{k}\right)$ by $\alpha_{i}(x)=\alpha_{i}^{*}\left(x_{i}\right)$.

THEOREM 6.4. For any positive integer $n$, the restriction of $L^{n}$ to $C_{0}^{\infty}\left(R^{k}\right)$ is essentially selfadjoint.

THEOREM 5.1. Let $\omega=\exp \left(\sum_{i=1}^{k} \alpha_{i}\right)$. Then for all solutions to $L f=\lambda f$ such that $f \in L_{2}\left(R^{k}\right)$, where $\lambda$ is a real number, it follows that $\omega f \in L_{2}\left(R^{k}\right)$. Furthermore, if $L f=\lambda f$ for some complex number $\lambda$, and $f \notin L_{2}\left(R^{k}\right)$, then $w^{-1} f \notin L_{2}\left(R^{k}\right)$.

THEOREM 6.6. Let $L$ be as in Definition 2.1, and assume in addition that

(i) $\alpha_{i}=o\left(p_{0}^{2}\right)$ for all $i$;

(ii) for each $i \in 1, \ldots, k$ there exists a $j(i)>0$ and a positive integer $n$ such that $p_{0}^{n} p_{i j(i)}$ is bounded away from zero;

(iii) $|x|^{\delta}=O\left(p_{0}\right)$ for some $\delta>0$.

Then, for any $\lambda$ with positive real part, the abstract Cauchy problem $d u(t) / d t=$ $-\lambda L u(t), u(0)=f$ is well posed and $\rho$-contractive in $L_{2, \rho}\left(R^{k}\right)$, where $\rho=\omega^{-2}$, and $\omega$ is as in the preceding theorem.

ExAmple 2.3. Let $L=-D\left(x^{2}+1\right)^{\alpha} D+\left(x^{2}+1\right)^{\beta}$, where $D=d / d x$. Suppose that $\alpha<\beta+1$, and that $\beta>0$. Then

(i) $L$ satisfies the hypotheses of Theorem 6.6 with $\omega=\exp \left(x^{2}+1\right)^{-\delta+(\beta-\alpha+1) / 2}$ for any positive $\delta$ such that $\delta<(\beta-\alpha+1) / 2$ if $\alpha>1-\beta$;

(ii) $L$ satisfies the hypotheses of Theorem 6.6 with $\omega=\exp \left(x^{2}+1\right)^{\beta-\delta}$ for all positive $\delta<\beta$ if $1-\beta \geq \alpha$.

3. Essential selfadjointness for $L$. In this section we show that the restriction of $L$ to $C_{0}^{\infty}\left(R^{k}\right)$ is essentially selfadjoint, where $L$ is as in Definition 2.1. The method of proof is that used by the author in [8], although that situation is more difficult because the higher order terms may be dominant. Although the situation of this paper is essentially much simpler, it is still necessary to give a new proof because the coefficients of this paper have a somewhat more general form. Where it does not seem to cause undue difficulty, we shall outline the argument here, referring the reader to the appropriate place in [8] for the details. 
LEMMA 3.1. Let $H$ be the Friedrichs extension in $L_{2}\left(R^{k}\right)$ of $T_{0}(L)$, where $T_{0}(L)$ denotes the closure of the restriction of $L$ to $C_{0}^{\infty}\left(R^{k}\right)$. Then $H^{-1}$ is compact.

PROOF. Note that since $T_{0}(L)$ is positive definite and bounded away from zero, $(H+\lambda I)^{-1}$ exists for all $\lambda \geq 0$. The assertion follows immediately from the fact that the embedding of $H^{1}(\Omega)$ into $L_{2}(\Omega)$ is compact, where $\Omega$ is a ball of radius $n$, by a well-known argument due to Rellich. The essence of the argument is that $H^{-1}$ is the limit in operator norm of operators $\theta_{n} H^{-1}$, where $\theta_{n} \in C_{0}^{\infty}\left(R^{k}\right),\left|\theta_{n}(x)\right| \leq 1$, $\theta_{n}(x)=1$ when $|x| \leq n$ and $\theta_{n}(x)=0$ when $|x| \geq n+1$. The operators $\theta_{n} H^{-1}$ are compact by the first assertion of the proof.

LEMMA 3.2. Suppose $p \in C^{\infty}\left(R^{k}\right)$, with $p(x)>0$ for all $x$. Suppose that $\varphi^{*} \in C^{\infty}\left(R^{1}\right)$, with $\varphi^{*(i)}=O\left(\left(\varphi^{*}\right)^{i+1}\right)$ for all $i \geq 1$, and with $\varphi^{*}>0$. Let $\varphi(x)$ be the element of $C^{\infty}\left(R^{1}\right)$ defined by $\varphi(x)=\varphi^{*}\left(x_{i}\right)$, and suppose that $D_{i}^{r}(p)=O\left(p \varphi^{r}\right)$ for all $r \geq 1$. Then there exists a $K>0$ such that for every $\varepsilon$ with $1 \geq \varepsilon>0$ and positive integers $r$ and $j$ such that $j \geq 2$ and $1 \leq r \leq j-1$, and for all $f$ in $C_{0}^{\infty}\left(R^{k}\right)$, then

$$
K\left(\varepsilon^{r}\left\|p D_{i}^{j} f\right\|+\varepsilon^{r-j}\left\|\varphi^{j} p f\right\|\right) \geq\left\|p \varphi^{r} D_{i}^{j-r} f\right\| .
$$

PROOF. It follows from integration by parts, as in the proof of [8, Lemma 1.10], that the above is true when $j=2$ and $r=1$. We complete the proof by induction.

Suppose there exists a $K$ such that for every $\delta$ with $0<\delta \leq 1$ and all $f$ in $C_{0}^{\infty}\left(R^{k}\right)$

$$
K\left(\delta^{r}\left\|p D_{i}^{n} f\right\|+\delta^{r-n}\left\|\varphi^{n} p f\right\|\right) \geq\left\|p \varphi^{r} D_{i}^{n-r} f\right\|
$$

for all $n \leq N$ and $r$ with $1 \leq r \leq n-1$. Then

$$
K\left(\delta^{r}\left\|p D_{i}^{N+1} f\right\|+\delta^{r-N}\left\|\varphi^{N} p D_{i} f\right\|\right) \geq\left\|p \varphi^{r} D_{i}^{N-r+1} f\right\|
$$

for all $r$ with $1 \leq r \leq N-1$. But, if $j \geq 1$, since $D_{i}^{r}\left(\varphi^{j-1} p\right)=O\left(\varphi^{j-1+r} p\right)$, it follows from the induction hypothesis that

$$
\left\|\varphi^{N} p D_{i} f\right\| \leq K_{1}\left(\left\|\delta_{1} \varphi^{N-1} D_{i}^{2} f\right\|+\left(1 / \delta_{1}\right)\left\|p \varphi^{N+1} f\right\|\right) .
$$

Setting $r=N-1$ in inequality (3.1), we see that

$$
K\left(\delta^{N-1}\left\|p D_{i}^{N+1} f\right\|+\delta^{-1}\left\|\varphi^{N} p D_{i} f\right\|\right) \geq\left\|p \varphi^{N-1} D_{i}^{2} f\right\| .
$$

Selecting $\delta_{1}=\delta / 2 K_{1} K$, and substituting (3.3) into (3.2) we see that

$$
K_{3} \delta^{N}\left\|p D_{i}^{N+1} f\right\|+\frac{1}{2}\left\|\varphi^{N} p D_{i} f\right\|+\left(2 K_{1}^{2} K / \delta\right)\left\|p \varphi^{N+1} f\right\| \geq\left\|p \varphi^{N} D_{i} f\right\| .
$$

The required inequality is proved for $j=N+1$ and $r=N$ :

$$
K\left(\delta^{N}\left\|p D_{i}^{N+1} f\right\|+\delta^{-1}\left\|p \varphi^{N+1} f\right\|\right) \geq\left\|p \varphi^{N} D_{i} f\right\| .
$$

Substituting (3.4) into (3.1), we easily complete the proof.

THEOREM 3.3. Let $L$ be as in Definition 1.1. Then the restriction of $L$ to $C_{0}^{\infty}\left(R^{k}\right)$ is essentially selfadjoint.

PROOF. The proof follows a line of argument introduced by the author in [8], except that in that paper the argument is considerably more difficult because $p_{0}^{2}$ is not necessarily the largest term of $L$. 
Define a quadratic form $F$ on $C_{0}^{\infty}\left(R^{k}\right)$ by $F(f, g)=\left(p_{0}^{2} L f, g\right)$. We study $F$ in detail; some of the inequalities used in this proof will also be needed later on in the paper. Note that $\left(p_{0}^{2} D_{i}^{j} p_{i j}^{2} D_{i}^{j} f, f\right)=\left\|p_{i j} p_{0} D_{i}^{j} f\right\|^{2}+E(f, f)$, where $E(f, f)$ is a sum of multiples of terms of type $\left(a D_{i}^{j-r} f, b D_{i}^{j-s} f\right)$, where $a=O\left(p_{i j} p_{0} \varphi_{i}^{r}\right)$ and $b=O\left(p_{i j} p_{0} \varphi_{i}^{s}\right)$. By Lemma 3.2, we see that for every $\varepsilon>0$ there exists a $K>0$ such that

$$
|E(f, f)|<\varepsilon\left\|p_{i j} p_{0} D_{i}^{j} f\right\|^{2}+K\left\|p_{i j} p_{0} \varphi_{i}^{j} f\right\|^{2} .
$$

But

$$
\left\|p_{i j} p_{0} \varphi_{i}^{j} f\right\|^{2} \leq \varepsilon\left\|p_{0}^{2} f\right\|^{2}+K\|f\|^{2}
$$

Hence

$$
|E(f, f)| \leq \varepsilon\left(\left\|p_{i j} p_{0} D_{i}^{j} f\right\|^{2}+\left\|p_{0}^{2} f\right\|^{2}\right)+K\|f\|^{2} .
$$

From inequality (3.5) together with inequality (3.3), we see that, if $K$ is large enough,

$$
\operatorname{Re} F(f, f)+K(f, f) \geq(1-\varepsilon)\|f\|_{G}^{2},
$$

where $\|f\|_{G}^{2}$ is defined by

$$
\|f\|_{G}^{2}=\sum_{i=1}^{k} \sum_{j=1}^{N(i)} \sum_{l=0}^{j}\left\|p_{i j} p_{0} \varphi_{i}^{l} D_{i}^{j-l} f\right\|^{2}+\left\|p_{0}^{2} f\right\|^{2} .
$$

It follows that $F+K$ is a sectorial form. Let $G$ denote the $m$-sectorial operator associated with the form $F+K$. It follows as in the proof of Lemma 1.27 of [8] that for $f \in$ domain $G$, with $G f \in C_{0}^{\infty}\left(R^{k}\right)$, then for any $\theta$ in $C_{0}^{\infty}\left(R^{k}\right)$ and any multi-index $\alpha, \theta D^{\alpha} f \in h_{G}$, where $h_{G}$ denotes the closure in the norm \|\|$_{G}$ of $C_{0}^{\infty}\left(R^{k}\right)$. We outline the argument here, referring the reader to [8] for the details of the argument. then

The key idea of the proof is to note that if $G f \in C_{0}^{\infty}\left(R^{k}\right)$, and $g \in C_{0}^{\infty}\left(R^{k}\right)$,

$$
\left|F\left(\theta \delta_{h}^{i} f, g\right)+F\left(f, \theta \delta_{-h}^{i} g\right)\right| \leq K\|f\|_{G}\|g\|_{G},
$$

where

$$
\delta_{h}^{i} f(x)=(f(x+h e(i))-f(x)) / h,
$$

where $e(i)$ is the vector in $R^{k}$ given by $e(i)(j)=\delta_{i}^{j}$. One then notes that, because of the above inequality, $\left|F\left(\theta \delta_{h}^{i} f, g\right)\right| \leq K_{1}\|g\|_{G}$. But it is easy to see that $\theta \delta_{h}^{i} f \in h_{G}$. Hence, selecting a sequence $g_{n}$ which converges to $\theta \delta_{h}^{i} f$ in $h_{G}$, we see that $\left\|\theta \delta_{h}^{i} f\right\|_{G}$ is bounded uniformly, independent of $h$. Letting $h(n)=1 / n$, we see that there exists a subsequence $h(n(j))$ converging to zero such that $\theta \delta_{h(n(j))}^{i} f$ converges weakly to an element $\gamma$ of the Hilbert space $h_{G}$. By weak continuity of the injection from $h_{G}$ into $L_{2}$, we see that this sequence also converges weakly in $L_{2}$. But it is evident that for any $g$ in $C_{0}^{\infty}\left(R^{k}\right),\left(\theta \delta_{h(n(j))}^{i} f, g\right)$ converges to $\left(-f, D_{i}(\theta g)\right)$. Hence $\theta D_{i} f=\gamma$, where the derivative is taken in the sense of distribution theory. It follows that $\theta D_{i} f \in h_{G}$. The argument proceeds by induction, following the proofs of Lemmas 1.26 and 1.27 of [8]. In this fashion, we see that $\theta D^{\alpha} f \in h_{G}$ for all $\alpha$.

We now show that if $\alpha$ is any multi-index, and $\varphi^{-\alpha}$ denotes $\varphi_{1}^{-\alpha(1)} \cdots \varphi_{k}^{-\alpha(k)}$, then $\varphi^{-\alpha} D^{\alpha} f \in h_{G}$ for all $f$ in the domain of $G$ such that $G f \in C_{0}^{\infty}\left(R^{k}\right)$. 
Let $\theta \in C_{0}^{\infty}\left(R^{1}\right)$, with $\theta(x)=1$ for $|x|=1$ and $\theta(x)=0$ for $|x| \geq 2$. Let $\theta_{n, i}(x)=\theta\left(x_{i} / n\right)$, and let $\xi_{n}(x)=\prod_{i=1}^{k} \theta_{n, i}(x)$. Note that $\left(x_{i}^{2}+1\right)^{-1 / 2}=O\left(\varphi_{i}\right)$, as is easily seen from differentiating $\varphi_{i}^{-1}$. Thus, for any $\alpha, D^{\alpha} \xi_{n} \leq K \varphi^{\alpha}$, where $K$ is independent of $n$. We prove an assertion we call Assertion A.

Assertion A. Suppose $f \in \operatorname{domain} G$, and $G f \in C_{0}^{\infty}\left(R^{k}\right)$. Suppose that for all $\beta$ with $|\beta| \leq N-1, \varphi^{-\beta} D^{\beta} f \in h_{G}$. Suppose $g \in C_{0}^{\infty}\left(R^{k}\right)$, and $|\alpha|=N \geq 1$. Then

$$
\begin{aligned}
& \left|F\left(\xi_{n} \varphi^{-\alpha} D^{\alpha} f, g\right)-(-1)^{|\alpha|} F\left(f, \xi_{n} \varphi^{-\alpha} D^{\alpha} g\right)\right| \\
& \quad \leq K \sum_{|\beta| \leq N-1}\left\|\varphi^{-\beta} D^{\beta} f\right\|_{G}\|g\|_{G} .
\end{aligned}
$$

Proof of Assertion A. Note that, for $h \in h_{G}$ and $g \in C_{0}^{\infty}\left(R^{k}\right), F(h, g)$ is a sum of terms of type $(\gamma f, \delta g)$, where $\gamma$ and $\delta$ are of the form $p_{0} \eta \varphi_{i}^{l} p_{i j} D_{i}^{j-l}$, for some $l$ with $0 \leq l \leq j$, where $\eta$ is bounded and $D^{\gamma} \eta=O\left(\varphi^{\gamma}\right)$ for all $\gamma$. It is clear that the expression $\gamma \xi_{n} \varphi^{-\alpha} D^{\alpha}-\xi_{n} \varphi^{-\alpha} D^{\alpha} \gamma$ is a sum of terms of the form $p_{0} \eta_{1} \varphi^{-\alpha+\tau} p_{i j} \varphi_{i}^{l} D^{\alpha-\tau} D_{i}^{j-l}$, where $\eta_{1}$ is bounded and $D^{\beta} \eta_{1}=O\left(\varphi^{\beta}\right)$ for all $\beta$. But $|\alpha-\tau| \leq N-1$. It follows from inequality (3.7) that, for $f$ as above,

$$
\left\|\left(\gamma \xi_{n} \varphi^{-\alpha} D^{\alpha}-\xi_{n} \varphi^{-\alpha} D^{\alpha} \gamma\right) f\right\| \leq K \sum_{|\beta| \leq N-1}\left\|\varphi^{-\beta} D^{\beta} f\right\|_{G}\|g\|_{G} .
$$

Similarly, integration by parts shows that

$$
\left|\left(\gamma f,\left(D^{\alpha} \xi_{n} \varphi^{-\alpha} \delta-\delta D^{\alpha} \xi_{n} \varphi^{-\alpha}\right) g\right)\right| \leq K \sum_{|\beta| \leq N-1}\left\|\varphi^{-\beta} D^{\beta} f\right\|_{G}\|g\|_{G}
$$

Assertion A is proved.

But $\left|F\left(f, \xi_{n} \varphi^{-\alpha} D^{\alpha} g\right)\right|=\left|\left(G f, \xi_{n} \varphi^{-\alpha} D^{\alpha} g\right)\right| \leq K\|g\|_{2}$. Letting $g_{j}$ converge in $h_{G}$ to $\xi_{n} \varphi^{-\alpha} D^{\alpha} f$, which was shown to be in $h_{G}$ by the first part of the proof, we see that $\left\|\xi_{n} \varphi^{-\alpha} D^{\alpha} f\right\|_{G} \leq K$ where $K$ is independent of $n$. Hence, once again, a subsequence of $\xi_{n} \varphi^{-\alpha} D^{\alpha} f$ converges weakly to an element $h$ of $h_{G}$; hence this convergence also occurs weakly in $L_{2}\left(R^{k}\right)$. It is evident that for any $g$ in $C_{0}^{\infty}\left(R^{k}\right)$, $\left(\xi_{n} \varphi^{-\alpha} D^{\alpha} f, g\right)$ converges to $\left(\varphi^{-\alpha} D^{\alpha} f, g\right)$; hence $\varphi^{-\alpha} D^{\alpha} f \in h_{G}$, as we desired to show.

From the fact that $\varphi^{-\alpha} D^{\alpha} f \in h_{G}$ for all $\alpha$, it follows that $p_{0} p_{i j} \varphi_{i}^{r} D_{i}^{2 j-r} f \in$ $L_{2}\left(R^{k}\right)$ for all $r \leq 2 j$. It is then clear that $L\left(\xi_{n} f\right)$ converges in $L_{2}\left(R^{k}\right)$ to $L f$ as $n$ approaches infinity; hence $f$ is in the domain of the closure $T_{0}(L)$ of the restriction of $L$ to $C_{0}^{\infty}\left(R^{k}\right)$.

Now we are able to complete the proof of the theorem. Suppose that $T_{0}(L)$ is not selfadjoint. Then, since the range of $T_{0}(L)$ is closed, it follows that the range of $T_{0}(L)$ is not dense in $L_{2}\left(R^{k}\right)$. In particular, there exists an $f$ in $C_{0}^{\infty}\left(R^{k}\right)$ such that $f$ is not in the range of $T_{0}(L)$. Let $\left(p_{0}^{2}+K\right) h=g$. By the definition of $G, G$ is surjective, so that there exists an element $f$ of domain $G$ such that $(G+K) f=g$. By the previous part of the proof, $f$ is in the domain of $T_{0}(L)$. Since $F(f, \alpha)=\left(p_{0}^{2} L f, \alpha\right)$ for all $\alpha \in C_{0}^{\infty}\left(R^{k}\right)$, it follows that $\left(p_{0}^{2}+K\right) L f=g=\left(p_{0}^{2}+K\right) h$. Hence $L f=h$, a contradiction. The theorem is proved.

4. Properties of $\omega L \omega^{-1}$. In this section we develop the properties of $\omega L \omega^{-1}$ which we need for two purposes. We must show that the closure $T_{0}\left(\omega L \omega^{-1}\right)$ of the restriction of $\omega L \omega^{-1}$ to $C_{0}^{\infty}\left(R^{k}\right)$ is $m$-sectorial, in order to be able to apply 
Theorem 1.4. We also show that $T_{0}\left(\omega L \omega^{-1}\right)$ is a relatively bounded perturbation of $T_{0}(L)$, where the difference has relative bound zero. We need this fact to analyze the growth and decay of eigenfunctions of $L$. Both these facts depend upon the calculation of the domain of $T_{0}(L)$; the second fact will be used to prove the first fact. For the function $\omega$ which we shall use for $L, \omega L \omega^{-1}$ will be of the form of Theorem 4.2.

LEMMA 4.1. There exists an $\varepsilon>0$ and a $K>0$ such that for all $f$ in $C_{0}^{\infty}\left(R^{k}\right)$,

$$
\begin{aligned}
\|(L+K) f\| \geq \varepsilon\left(\sum_{i, r=1, \ldots, k} \sum_{j=1}^{N(i)} \sum_{s=1}^{N(r)}\left\|p_{i j} p_{r s} D_{i}^{j} D_{r}^{s} f\right\|\right. & \\
& \left.+\sum_{i=1}^{k} \sum_{j=1}^{N(i)}\left\|p_{0} p_{i j} D_{i}^{j} f\right\|+\left\|p_{0}^{2} f\right\|\right) .
\end{aligned}
$$

Proof. $(L f, L f)=\left(L^{2} f, f\right) \cdot\left(L^{2} f, f\right)$ is a sum of terms of type

$$
\left((-1)^{i+s} D_{i}^{j} p_{i j}^{2} D_{i}^{j} D_{r}^{s} p_{r s}^{2} D_{r}^{s} f, f\right),
$$

together with terms of type $(-1)^{j}\left(p_{0}^{2} D_{i}^{j} p_{i j}^{2} D_{i}^{j} f, f\right)$ and $\left(p_{0}^{4} f, f\right)$. But, by inequality (3.5), for every $\delta>0$ there exists a $K>0$ such that

$$
\begin{aligned}
(-1)^{j} & \operatorname{Re}\left(p_{0}^{2} D_{i}^{j} p_{i j}^{2} D_{i}^{j} f, f\right)+\left(p_{0}^{4} f, f\right)+K(f, f) \\
& \geq(1-\delta)\left(\left\|p_{i j} p_{0} D_{i}^{j} f\right\|^{2}+\left\|p_{0}^{2} f\right\|^{2}\right) \\
& \geq(1-2 \delta)\left(\sum_{l=0}^{j}\left\|p_{i j} p_{0} \varphi_{i}^{l} D_{i}^{j-l} f\right\|^{2}+\left\|p_{0}^{2} f\right\|^{2}\right)-K_{1}\|f\|^{2},
\end{aligned}
$$

where the last inequality follows from Lemma 3.2.

If $i=r$, Lemma 3.2 guarantees that

$$
(-1)^{j+s}\left(D_{i}^{j} p_{i j}^{2} D_{i}^{j} D_{r}^{s} p_{r s}^{2} D_{r}^{s} f, f\right)=\left\|p_{i j} p_{i s} D_{i}^{j+s} f\right\|^{2}+E(f, f),
$$

where for every $\delta>0$ there exists a $K>0$ such that

$$
\begin{aligned}
|E(f, f)| & \leq \delta\left\|p_{i j} p_{i s} D_{i}^{j+s} f\right\|^{2}+K\left\|p_{i j} p_{i s} \varphi_{i}^{r+s} f\right\|^{2} \\
& \leq \delta\left(\left\|p_{i j} p_{i s} D_{i}^{j+s} f\right\|^{2}+\left\|p_{0}^{2} f\right\|^{2}\right)+K_{2}\|f\|^{2} .
\end{aligned}
$$

If $i \neq r$, Lemma 3.2 shows that

$$
(-1)^{j+s}\left(D_{i}^{j} p_{i j}^{2} D_{i}^{j} D_{r}^{s} p_{r s}^{2} D_{r}^{s} f, f\right)=\left\|p_{i j} p_{r s} D_{i}^{j} D_{r}^{s} f\right\|^{2}+E(f, f),
$$

where $E(f, f)$ is a sum of terms of type $\left(a D_{i}^{j-l} D_{r}^{s-m} f, b D_{i}^{j-n} D_{r}^{s-p} f\right)$, with $a=$ $O\left(p_{i j} p_{r s} \varphi_{i}^{l} \varphi_{r}^{m}\right)$ and $b=O\left(p_{i j} p_{r s} \varphi_{i}^{n} \varphi_{r}^{p}\right)$, and where $l, m, n$ and $p$ are not all zero. By Lemma 3.2, if $l \neq 0$, then for every $\varepsilon>0$ there exists a $K>0$ such that

$$
\left\|a D_{i}^{j-l} D_{r}^{s-m} f\right\| \leq \delta\left\|p_{i j} p_{r s} \varphi_{r}^{m} D_{i}^{j} D_{r}^{s-m} f\right\|+K_{3}\left\|p_{i j} \varphi_{i}^{j} p_{r s} \varphi_{r}^{m} D_{r}^{s-m} f\right\| .
$$

If $m \neq 0$,

$$
\left\|p_{i j} p_{r s} \varphi_{r}^{m} D_{i}^{j} D_{r}^{s-m} f\right\| \leq \delta\left\|p_{i j} p_{r s} D_{i}^{j} D_{r}^{s} f\right\|+K_{4}\left\|p_{i j} p_{r s} \varphi_{r}^{s} D_{i}^{j} f\right\|,
$$

and

$$
K_{3}\left\|p_{i j} \varphi_{i}^{j} p_{r s} \varphi_{r}^{m} D_{r}^{s-m} f\right\| \leq \delta\left\|p_{i j} \varphi_{j}^{i} p_{r s} D_{r}^{s} f\right\|+K_{5}\left\|p_{i j} p_{r s} \varphi_{i}^{j} \varphi_{r}^{s} f\right\|
$$


But

$$
K_{5}\left\|p_{i j} p_{r s} \varphi_{i}^{j} \varphi_{r}^{s} f\right\|<\delta\left\|p_{0}^{2} f\right\|+K_{6}\|f\| .
$$

We have thus seen that, if $i \neq r$,

$$
(-1)^{j+s}\left(D_{i}^{j} p_{i j}^{2} D_{i}^{j} D_{r}^{s} p_{r s}^{2} D_{r}^{s} f, f\right)=\left\|p_{r s} p_{i j} D_{r}^{s} D_{i}^{j} f\right\|^{2}+E(f, f)
$$

where for every $\delta>0$ there exists a $K>0$ and $K_{1}>0$ such that

$$
\begin{aligned}
|E(f, f)| \leq & \delta\left(\left\|p_{i j} p_{r s} D_{r}^{s} D_{i}^{j} f\right\|^{2}+\left\|p_{0}^{2} f\right\|^{2}\right) \\
& +K\left(\left\|p_{i j} p_{r s} \varphi_{r}^{s} D_{i}^{j} f\right\|^{2}+\left\|p_{i j} p_{r s} \varphi_{i}^{j} D_{r}^{s} f\right\|^{2}\right)+K_{1}\|f\|^{2} .
\end{aligned}
$$

However,

$$
\left\|p_{i j} p_{r s} \varphi_{r}^{s} D_{i}^{j} f\right\|^{2} \leq(\delta / 2 K)\left\|p_{0} p_{i j} D_{i}^{j} f\right\|^{2}+K_{2}\left\|p_{i j} D_{i}^{j} f\right\|^{2}
$$

and

$$
\left\|p_{i j} p_{r s} \varphi_{i}^{j} D_{r}^{s} f\right\|^{2} \leq(\delta / 2 K)\left\|p_{0} p_{r s} D_{r}^{s} f\right\|^{2}+K_{3}\left\|p_{r s} D_{r}^{s} f\right\|^{2} .
$$

The conclusion follows from inequalities (4.1), (4.2) and (4.3).

THEOREM 4.2. Suppose that $M=L+\sum_{i=1}^{k} \sum_{1 \leq j<2 N(i)} \alpha_{i j} D_{i}^{j}+\alpha_{0}$, where $\alpha_{i j} \in C^{\infty}\left(R^{k}\right), \alpha_{0} \in C^{\infty}\left(R^{k}\right)$, and

(a) for all integers $t$ such that $0 \leq t<j, D_{i}^{t}\left(\alpha_{i j}\right)=O\left(\sum_{r>j / 2} p_{i r}^{2} \varphi_{i}^{2 r-j+t}\right)$;

(b) $\alpha_{0}=o\left(p_{0}^{2}\right)$.

Then

(i) the restriction $R$ of $M$ to the domain of $T_{0}(L)$ is $m$-sectorial;

(ii) $R=T_{0}(M)$, where $T_{0}(M)$ denotes the closure of the restriction of $M$ to $C_{0}^{\infty}\left(R^{k}\right)$

(iii) $T_{0}(M)$ is the restriction of $M$ to $\left\{f \in L_{2}\left(R^{k}\right) \mid M f \in L_{2}\left(R^{k}\right)\right\}$, where the derivatives are taken in the distribution sense;

(iv) for all complex $\lambda, R-\lambda$ is a Fredholm operator;

(v) the Fredholm index of $R-\lambda$ is zero for all $\lambda$.

ProOF. Select $K$ as in Lemma 4.1. Note that

$$
\|(M+K) f\|^{2}=\|(L+K) f\|^{2}+E(f, f)
$$

for all $f$ in $C_{0}^{\infty}\left(R^{k}\right)$, where $E(f, f)$ is a sum of terms of the form $\left(\alpha_{i j} D_{i}^{j} f,(L+K) f\right)$ and terms of the form $\left(\alpha_{i j} D_{i}^{j} f, \alpha_{r s} D_{r}^{s} f\right)$.

Clearly, for some positive constant $K_{0},|E(f, f)| \leq K_{0}\|(L+K) f\|\left\|\alpha_{i j} D_{i}^{j} f\right\|$. By hypothesis, and Lemmas 3.2 and 4.1 , for every $\varepsilon>0$ there exists a $K_{1}>0$ such that $\left\|\alpha_{i j} D_{i}^{j} f\right\| \leq \varepsilon\|(L+K) f\|+K_{1} \sum_{s>j}\left\|p_{i s}^{2} \varphi_{i}^{2 s} f\right\|$. Clearly,

$$
K_{1} \sum_{s>j}\left\|p_{i s}^{2} \varphi_{i}^{2 s} f\right\| \leq \varepsilon\left\|p_{0}^{2} f\right\|+K_{2}\|f\| \leq \varepsilon\|(L+K) f\|+K_{1}\|f\| .
$$

Hence, we see that

$$
|E(f, f)| \leq 2 \varepsilon\|(L+K) f\|^{2}+K_{1}\|f\|\|(L+K) f\| \leq 3 \varepsilon\|(L+K) f\|^{2}+K_{2}\|f\|^{2} .
$$

Hence the restriction of $M-L$ to the domain of $T_{0}(L+K)$ is relatively bounded, with relative bound zero. It follows that the restriction of $M$ to the domain of $T_{0}(L)$ is a closed operator. Hence, if $T_{0}(M)$ is defined to be the closure of the restriction 
of $M$ to $C_{0}^{\infty}\left(R^{k}\right)$, it is clear that the restriction of $M$ to the domain of $T_{0}(L)$ contains $T_{0}(M)$. However, it is evident from Lemmas 4.1 and 3.3 that for any $f$ in the domain of $T_{0}(L), L\left(\xi_{n} f\right)$ converges in $L_{2}\left(R^{k}\right)$ to $L f$, where $\xi_{n}$, as in the proof of Theorem 3.3, denotes $\prod_{i=1}^{k} \theta\left(x_{i} / n\right)$, with $\theta \in C_{0}^{\infty}\left(R^{1}\right)$ such that $\theta(x)=0$ for $|x| \geq 2$ and $\theta(x)=1$ for $|x| \leq 1$. It is also clear from regularization that $\xi_{n} f$ is in the domain of $T_{0}(M)$. Hence, $T_{0}(M)$ is the restriction of $M$ to the domain of $T_{0}(L)$.

It is evident from integration by parts together with Lemma 3.2 that for every $\varepsilon>0$ there exists a $K>0$ such that

$$
\begin{aligned}
\operatorname{Re}((M-L) f, f) & \leq \varepsilon(L f, f)+K\left(\sum_{i=1}^{k} \sum_{j>0}\left\|p_{i j} \varphi_{i}^{j} f\right\|^{2}+1\right) \\
& \leq \varepsilon\left((L f, f)+\left\|p_{0} f\right\|^{2}\right)+K_{1}\|f\|^{2} .
\end{aligned}
$$

It follows that $T_{0}(M)$ is bounded below.

Let $L_{\varepsilon}=L+\varepsilon(M-L)$. From the above, together with inequality 4.1, we see that $T_{0}\left(L_{\varepsilon}\right)$ is bounded below, and that $T_{0}\left(L_{\varepsilon}\right)$ is the restriction of $L_{\varepsilon}$ to the domain of $T_{0}(L)$. From inequality (4.1) we see that for $K$ large, depending (perhaps) on $\varepsilon, \operatorname{Re}((M+K) f, f) \geq \varepsilon(L f, f)$. Hence, just as was the case for the Friedrichs extension of $T_{0}(L)$, approximation by compact operators shows that the Friedrichs extension $H_{M}$ of $T_{0}(M)$ is a discrete operator, in the sense that $\left(H_{M}+K\right)^{-1}$ is compact for some constant $K$.

We now show that $T_{0}\left(L_{\varepsilon}\right)$ is $m$-sectorial. Note that since $H_{M}+K$ has a compact resolvent for some $K$, it follows that $H_{M}$ is a Fredholm operator. Also, by Theorem 2.5, p. 323, Kato [7], $\left(H_{M}+K\right)^{*}$ is the Friedrichs extension of $T_{0}\left(M^{+}\right)$, where $M^{+}$ is the adjoint expression for $M$. But $M^{+}$is of the same form as $M$. Since, for $K$ large enough, $\operatorname{Re}((M+K) f, f) \geq(f, f)$ for all $f$ in $C_{0}^{\infty}\left(R^{k}\right)$, it follows from the definition of $H_{M}$ that $H_{M}+K$ is 1-1. Similarly, $\left(H_{M}+K\right)^{*}$ is 1-1. Thus the index of $H_{M}+K$ is zero. Since $K$ is relatively compact with respect to $H_{M}$, it follows that the index of $H_{M}$ is zero. If we can show that the index of $T_{0}(M)$ is zero, it will follow that $T_{0}(M)=H_{M}$.

Let $S=\left\{\varepsilon \mid\right.$ index $\left.T_{0}\left(L_{\varepsilon}\right)=0\right\}$. We show that $S$ is relatively open, closed and nonempty, and hence that $S=[0,1]$. We do this by showing that for any $a \in[0,1]$, there exists a relatively open neighborhood $\theta_{a}$ of $a$ in $[0,1]$ such that index $T_{0}\left(L_{\varepsilon}\right)=$ index $T_{0}\left(L_{a}\right)$ for all $\varepsilon$ in $\theta_{a}$. Select $K$ large enough that $\operatorname{Re}\left(\left(L_{\varepsilon}+K\right) f, f\right) \geq(f, f)$ for all $\varepsilon$ in some neighborhood of $a$. Then $T_{0}\left(L_{a}+K\right)$ is a semi-Fredholm operator, since it is 1-1. Furthermore, for all $\varepsilon$, the domain of $T_{0}\left(L_{\varepsilon}\right)$ is the same as the domain of $T_{0}(L)$. Clearly, for example by Theorem 5.22, p. 236, Kato [7], for all $\varepsilon$ in a neighborhood of $a$, the index of $T_{0}\left(L_{\varepsilon}+K\right)$ is the same as the index of $T_{0}\left(L_{a}+K\right)$. But by the relative compactness of the operator of multiplication by $K$, the index of $T_{0}\left(L_{\varepsilon}\right)+K$ is the same as the index of $T_{0}\left(L_{\varepsilon}\right)$. Furthermore, $T_{0}\left(L_{\varepsilon}\right)+K=T_{0}\left(L_{\varepsilon}+K\right)$. Hence $S$ is both relatively open and closed. $S$ is nonempty since index $T_{0}\left(L_{0}\right)=0$.

Finally, to see that the domain of $T_{0}(M)$ is $\left\{f \in L_{2}\left(R^{k}\right) \mid M f \in L_{2}\left(R^{k}\right)\right\}$, we note that $M^{+}$has the same form as $M$. Also, note that $T_{0}\left(M^{+}\right)^{*}$ is clearly the restriction $T_{1}(M)$ of $M$ to the above domain. However, since $T_{0}\left(M^{+}\right)$is its own Friedrichs extension, and since, as we saw earlier in the proof, $H_{M}^{*}$ is the Friedrichs 
extension of $T_{0}\left(M^{+}\right)$, it follows that $T_{0}(M)=H_{M}=T_{0}\left(M^{+}\right)^{*}=T_{1}(M)$. The theorem is proved.

5. Growth and decay of the eigenfunctions. In this section we compute the rate of growth and decay of the eigenfunctions of $L$. An example is instructive here. If $L=-D^{2}+x^{2}$, the $L_{2}$ eigenfunctions may be computed directly, and are known to be Hermite polynomials multiplied by $\exp \left(-x^{2} / 2\right)$. If we use the methods of this section on this example, we find that $\omega f_{n} \in L_{2}(R)$ for any such eigenfunction, where $\omega$ is a positive $C^{\infty}$ function which agrees with $\exp \left(|x|^{2-\varepsilon}\right)$ for large $|x|$. We also see that if $L f=\lambda f$, and $f \notin L_{2}(R)$, where $L=-D^{2}+x^{2}$, then $\omega^{-1} f \notin L_{2}(R)$. The above example gives some idea of the extremely rapid decay of the eigenfunctions of $L$, and also shows that our methods compute this decay rate fairly accurately.

THEOREM 5.1. Let $\alpha_{i}$ and $\omega$ be as in $\S 2$. Then

(a) for all $f$ in $L_{2}\left(R^{k}\right)$ such that $L f=\lambda f$ for some real $\lambda$, it follows that $\omega f \in L_{2}\left(R^{k}\right)$

(b) if $L f=\lambda f$ for some complex number $\lambda$, and $f \notin L_{2}\left(R^{k}\right)$, then $\omega^{-1} f \notin$ $L_{2}\left(R^{k}\right)$.

Proof. Let $M=\omega L \omega^{-1}$. Note that $M$ has the form of Theorem 4.2. Suppose that, for some real $\lambda, M f=\lambda f$, and $f \in L_{2}\left(R^{k}\right)$. Then $\omega^{-1} f \in L_{2}\left(R^{k}\right)$, and $L \omega^{-1} f=\lambda \omega^{-1} f$. Hence, the nullity of $T_{1}(L-\lambda)$ is greater than or equal to the nullity of $T_{1}(M-\lambda)$, where $T_{1}(M-\lambda)$ denotes the restriction of $M-\lambda$ to $\{f \in$ $\left.L_{2}\left(R^{k}\right) \mid(M-\lambda) f \in L_{2}\left(R^{k}\right)\right\}$, where the derivatives are taken in the distribution sense. Similarly, we see that, if $Q=\omega^{-1} L \omega$, the nullity of $T_{1}(Q-\lambda)$ is less than or equal to the nullity of $T_{1}(L-\lambda)$. But $Q$ and $M$ are of the form of Theorem 4.2; hence $T_{1}(Q-\lambda)$ and $T_{1}(M-\lambda)$ are Fredholm operators of index zero. However, if $T_{0}(M)$ denotes the closure of the restriction of $M$ to $C_{0}^{\infty}\left(R^{k}\right), T_{0}(M-\lambda)^{*}=T_{1}(Q-\lambda)$. But by Theorem 4.2, $T_{0}(M-\lambda)=T_{1}(M-\lambda)$, so $T_{1}(M-\lambda)^{*}=T_{1}(Q-\lambda)$. The index of $T_{1}(M-\lambda)$ is the nullity of $T_{1}(M-\lambda)$ minus the nullity of $T_{1}(Q-\lambda)$; Theorem 4.2 shows that this index is zero. Hence

$$
\text { nullity } T_{1}(M-\lambda)=\text { nullity } T_{1}(L-\lambda)=\text { nullity } T_{1}(Q-\lambda) \text {. }
$$

Furthermore, since $T_{1}(L)$ is a Fredholm operator, all of these nullities are finite.

Note that $\left\{\omega^{-1} g \mid g \in\right.$ null space $\left.T_{1}(M-L)\right\} \subseteq$ null space $T_{1}(L-\lambda)$. Since these two spaces have the same finite dimension, they are equal. Hence, if $L f=\lambda f$, with $f \in L_{2}\left(R^{k}\right)$, then $f=\omega^{-1} g$ for some $g \in L_{2}\left(R^{k}\right)$. (a) is proved.

Furthermore, the same argument shows that

$$
\left\{\omega^{-1} f \mid f \in \text { null space } T_{1}(L-\lambda)\right\}=\text { null space } T_{1}(Q-\lambda) \text {. }
$$

However, if $L h=\lambda h$, with $h \notin L_{2}\left(R^{k}\right)$ but with $\omega^{-1} h \in L_{2}\left(R^{k}\right)$, then $\omega^{-1} h$ would be in the null space of $T_{1}(Q-\lambda)$. This is a contradiction. (b) is proved.

LEMMA 5.2. For every $\varepsilon>0$, there exists a $K>0$ such that for all $\lambda$ in the spectrum of $T_{0}(L)$, and all solutions $f$ in $L_{2,1 / \rho}\left(R^{k}\right)$ to the equation $L f=\lambda f$, then $\|f\|_{1 / \rho} \leq K R(\lambda)\|f\|$, where \|\|$_{1 / \rho}$ is the norm of $L_{2,1 / \rho}\left(R^{k}\right)$ and $R(\lambda)$ is the largest value of $\omega$ on $\left\{x \mid(1-\varepsilon) p_{0}^{2} \leq K+\lambda+1\right\}$.

PROOF. Let $M=\omega L \omega^{-1}$. Then $M(\omega f)=\lambda \omega f$, and by the preceding theorem $\omega f \in L_{2}\left(R^{k}\right)$. For any $M$ of the form of Theorem 4.2 , then for all $f$ in $C_{0}^{\infty}\left(R^{k}\right)$, 
$\left(\alpha_{i j} D_{i}^{j} f, f\right)$ is a sum of terms of type $\left(a D_{i}^{l} f, b D_{i}^{s} f\right)$, where $a=O\left(\sum_{r>j / 2} p_{i r} \varphi_{i}^{r-l}\right)$, $b=O\left(\sum_{r>j / 2} p_{i r} \varphi_{i}^{r-s}\right), l \leq j / 2$, and $s \leq j / 2$. Hence, by inequality (3.1), we see that, for all $f$ in $C_{0}^{\infty}\left(R^{k}\right),|(M f, f)-(L f, f)| \leq \varepsilon(L f, f)+K\|f\|^{2}$. Since $T_{1}(M)$ and $T_{1}(L)$ are the closure of their restrictions to $C_{0}^{\infty}\left(R^{k}\right)$, we see that for every $\varepsilon>0$ there exists a $K$ such that

$$
(M(\omega f), \omega f) \geq(1-\varepsilon)(L(\omega f), \omega f)-K\|\omega f\|^{2} \geq(1-\varepsilon)\left\|p_{0} \omega f\right\|^{2}-K\|\omega f\|^{2} .
$$

Hence if $f \in L_{2}\left(R^{k}\right)$ and $L f=\lambda f,(\lambda+K)\|\omega f\|^{2} \geq(1-\varepsilon)\left\|p_{0} \omega f\right\|^{2}$. But if $\left\{x \mid(1-\varepsilon) p_{0}^{2} \leq K+1+\lambda\right\}$ is denoted by $\Omega(\lambda)$, and $\Gamma(\lambda)$ denotes $R^{k}-\Omega(\lambda)$,

$$
\int_{\Omega(\lambda)}\left((\lambda+K)-(1-\varepsilon) p_{0}^{2}\right)|\omega f|^{2} \geq \int_{\Gamma(\lambda)}|\omega f|^{2}
$$

Hence

$$
\|\omega f\|^{2} \leq(\lambda+K+1) \int_{\Omega(\lambda)}|\omega f|^{2} \leq(\lambda+K+1)(R(\lambda))^{2}\|f\|^{2} .
$$

The lemma is proved.

THEOREM 5.3. Suppose that, for every $i, \alpha_{i}=o\left(p_{0}^{2}\right)$, and that, if $\left\{\lambda_{n}\right\}$ is the spectrum of $T_{0}(L)$, listed in nondecreasing order according to multiplicity,

$$
\sum_{1}^{\infty} \exp \left(-\varepsilon \lambda_{n}\right)<\infty \quad \text { for every } \varepsilon>0 .
$$

Then, for any $\lambda$ such that $\operatorname{Re}(\lambda)>0$, the abstract Cauchy problem $d u(t) / d t=$ $-\lambda L u(t), u(0)=f$, is well posed and $\rho$-contractive in $L_{2, \rho}\left(R^{k}\right)$, where $\omega^{-2}=\rho$, with $\omega$ as in $\S 2$.

Proof. All of the hypotheses of Theorem 1.4 have been verified in previous results, except (iv). But $\left\|f_{n}\right\|_{1 / \rho}=\|\omega f\|$. By the previous lemma, $\left\|\omega f_{n}\right\| \leq$ $\left(\lambda_{n}+K+1\right)^{1 / 2} R\left(\lambda_{n}\right)$. But

$$
\ln R\left(\lambda_{n}\right) \leq \max \left\{\sum_{i=1}^{k} \alpha_{i}(x) \mid(1-\varepsilon) p_{0}^{2}(x) \leq \lambda_{n}+K+1\right\} .
$$

By hypothesis, $\sum_{i=1}^{k} \alpha_{i}(x)=o\left(p_{0}^{2}\right)$. Hence $\ln R\left(\lambda_{n}\right)=o\left(\lambda_{n}\right)$. But therefore $\ln \left(\lambda_{n} R\left(\lambda_{n}\right)\right)=o\left(\lambda_{n}\right)$. Hence $\lambda_{n} R\left(\lambda_{n}\right)=o\left(\exp \left(\varepsilon \lambda_{n}\right)\right)$ for all positive $\varepsilon$. Hence, for any positive $\delta, \exp \left(-\delta \lambda_{n}\right)\left\|\omega f_{n}\right\|=o\left(\exp \left(-\delta \lambda_{n} / 2\right)\right)$. Thus hypothesis (iv) of Theorem 1.4 holds; the theorem is proved.

6. Asymptotics of the spectrum. In this section, we show that, if $\lambda_{n}$ is as in Theorem 1.4, $\sum_{n=1}^{\infty} \exp \left(-\varepsilon \lambda_{n}\right)<\infty$ for every $\varepsilon>0$. This fact, together with Theorem 5.3, will suffice to prove Theorem 6.6, which was the goal of the paper. However, in order to prove the above spectral estimate, we shall need to know not only that the restriction of $L$ of $C_{0}^{\infty}\left(R^{k}\right)$ is essentially selfadjoint, but that the same conclusion is true for all powers of $L$. This fact is interesting in its own right. It is established without any additional hypotheses on $L$. However, to get the desired asymptotic behavior for $\lambda_{n}$, we shall need one additional hypothesis, which we set out below. Basically, this hypothesis states that not all of the higher order coefficients are excessively small relative to $p_{0}$. 
LEMMA 6.1. Let $R=p_{0}^{2 q} L^{n}$, where $q$ is a nonnegative integer and $n$ is a positive integer. Define a term of type I of $p_{0}^{2 q} L^{n}$ to be a term of type $(-1)^{|\alpha|} D^{\alpha} a_{\alpha}^{2} D^{\alpha}$, where $a_{\alpha}$ is a product of the form $p_{0}^{2 q+2(n-r)} \prod_{l=1}^{r} \xi_{l}$, $\xi_{l}$ is of the form $p_{i(l) j(l)}^{2}$, where the $\xi_{l}$ need not be distinct, and $\alpha=\sum_{l=1}^{r} j(l) e(i(l))$, where $e(i(l))$ is the $k$-tuple such that $e(i(l))_{m}=\delta_{i(l)}^{m}$, and $\delta$ is the Kronecker $\delta$. Define a term of type II of $p_{0}^{2 q} L^{n}$ to be a term of type $D^{\gamma} b_{\gamma \beta}^{2} D^{\beta}$, where $\gamma$ and $\beta$ are $k$-tuples of integers and $b_{\gamma \beta}^{2}=O\left(a_{\alpha}^{2} \varphi^{2 \alpha-\gamma-\beta}\right)$, where $(-1)^{|\alpha|} D^{\alpha} a_{\alpha}^{2} D^{\alpha}$, is a term of type I, and $\varphi^{2 \alpha-\gamma-\beta}=\prod_{i=1}^{k} \varphi_{i}^{2 \alpha(i)-\gamma(i)-\beta(i)}$, where $\gamma(i) \leq \alpha(i)$ for all $i, \beta(i) \leq \alpha(i)$ for all $i$, and $\gamma+\beta \neq 2 \alpha$. Then $R$ is a sum of terms of type I and terms of type II.

PROOF. The proof is obvious.

LEMMA 6.2. Let $R$ be as in the preceding lemma. Then for every $\varepsilon>0$ there exists a $K>0$ such that, if $D^{\alpha} b_{\gamma \beta} D^{\beta}$ is a term of type II of $R$,

$$
\left|\left(D^{\gamma} b_{\gamma \beta}^{2} D^{\beta} f, f\right)\right| \leq \varepsilon S_{n}+K S_{n-1},
$$

where $S_{n}=\sum\left\|a_{\alpha} D^{\alpha} f\right\|^{2}$, where the sum is taken over all terms of type I of $R$, and $S_{n-1}=\sum\left\|c_{\alpha} D^{\alpha} f\right\|^{2}$, where the second sum is taken over all terms of type I of $p_{0}^{2 q} L^{n-1}$, where $L^{n-1}=1$ if $n-1=0$.

ProOF. We use the notation of Lemma 6.1. $D^{\gamma} b_{\gamma \beta}^{2} D^{\beta}$ has associated with it, in the sense of Lemma 6.1, a term $(-1)^{|\alpha|} D^{\alpha} a_{\alpha}^{2} D^{\alpha}$ which is of type $I$ in $p_{0}^{2 q} L^{n}$. Let $\{i \mid \beta(i) \neq \alpha(i)\}$ be denoted by $T$. Let $Q=\{l \mid i(l) \in T\}$, and $\Gamma(Q)=$ cardinality $(Q)$. Lemma 3.2 shows that for every $\varepsilon>0$ there exists a $K>0$ such that

$$
\begin{aligned}
\left\|b_{\gamma \beta} D^{\beta} f\right\| \leq \varepsilon & \left\|p_{0}^{q+(n-r)} \prod_{l=1}^{r} p_{i(l) j(l)} D^{\alpha} f\right\| \\
& +K \sum_{S \subset T}\left\|\prod_{l=1}^{r} p_{i(l) j(l)} p_{0}^{q+n-r} \prod_{i(l) \in S} \varphi_{i(l)}^{j(l)} \prod_{i(l) \neq S} D_{i(l)}^{j(l)} f\right\|
\end{aligned}
$$

for all $f$ in $C_{0}^{\infty}\left(R^{k}\right)$, where the above sum is taken over all nonempty subsets $S$ of $T$. Note that if $t \notin T, \prod_{i(l)=t} D_{i(l)}^{j(l)}=D_{t}^{\alpha(t)}$.

However, for any $i(l) \in T, p_{i(l) j(l)} \varphi_{i(l)}^{j(l)}=o\left(p_{0}\right)$. Hence, for every $\varepsilon>0$,

$$
\begin{aligned}
& \left\|\prod_{l=1}^{r} p_{i(l) j(l)} p_{0}^{q+n-r} \prod_{l \in Q} \varphi_{i(l)}^{j(l)} \prod_{l \notin Q} D_{i(l)}^{j(l)} f\right\| \\
& \leq \varepsilon\left\|p_{0}^{q+n-r+\Gamma(Q)} \prod_{l \notin Q} p_{i(l) j(l)} \prod_{l \notin Q} D_{i(l)}^{j(l)} f\right\| \\
& \quad+K_{1}\left\|p_{0}^{q+n-r} \prod_{l \notin Q} p_{i(l) j(l)} \prod_{l \notin Q} D_{i(l)}^{j(l)} f\right\| .
\end{aligned}
$$

However

$$
\prod_{l \notin Q} D_{i(l)}^{j(l)} p_{0}^{2(q+\Gamma(Q)-1+n-r)} \prod_{l \notin Q} p_{i(l) j(l)}^{2} \prod_{l \notin Q} D_{i(l)}^{j(l)}
$$


is a term of type I of $p_{0}^{2 q} L^{n-1}$ and $\Gamma(Q)-1 \geq 0$ and $p_{0}$ is bounded away from zero. The lemma is proved.

LEMMA 6.3. There exists an $N>0$ such that, if $K_{i+1} / K_{i} \geq N$, where $\left\{K_{i}\right\}_{1}^{n}$ is a set of positive real numbers, then there exists an $\varepsilon>0$ such that for every $f$ in $C_{0}^{\infty}\left(R^{k}\right), \operatorname{Re}\left(p_{0}^{2 q} \prod_{i=1}^{n}\left(L+K_{i} f, f\right)\right) \geq \varepsilon S_{n}$, where $S_{n}$ is defined in Lemma 4.2.

PROOF. This follows from the preceding two lemmas.

THEOREM 6.4. The restriction of $L^{n}$ to $C_{0}^{\infty}\left(R^{k}\right)$ is essentially selfadjoint, for all $n \geq 1$.

PROOF. We have already seen that the assertion is true for $n=1$. We follow the line of argument of Theorem 3.3, using Lemma 6.3. Let $G$ be the Friedrichs extension of the restriction of $R=p_{0}^{2 n} \prod_{i=1}^{n}\left(L+K_{i}\right)$ to $C_{0}^{\infty}\left(R^{k}\right)$, which exists by Lemma 6.3.

Just as in Theorem 3.3, it may be seen that for any $f$ in the domain of $G$ such that $G f \in C_{0}^{\infty}\left(R^{k}\right)$, then $\varphi^{-\beta} D^{\beta} f \in h_{G}$, where $h_{G}$ is the completion of $C_{0}^{\infty}\left(R^{k}\right)$ in the norm $\|f\|_{G}^{2}=\sum\left\|a_{\alpha} D^{\alpha} f\right\|^{2}$, where the sum is taken over all terms of type I in $R$, where $\beta$ is any multi-index, and $\varphi^{-\beta}=\varphi_{1}^{-\beta(1)} \varphi_{1}^{-\beta(2)} \cdots \varphi_{k}^{-\beta(k)}$. However, this implies in particular that $p_{0}^{2 n} \varphi^{-\beta} D^{\beta} f \in L_{2}\left(R^{k}\right)$ for any such $\beta$.

Any term of $L^{n}$ is of the form $b_{\alpha} D^{\alpha}$, where

$$
b_{\alpha}=O\left(\prod_{l=1}^{r} p_{i(l) j(l)}^{2} \varphi_{i(l)}^{2 j(l)-\alpha(l)}\right) p_{0}^{2(n-r)} ;
$$

but $\prod_{l=1}^{r} p_{i(l) j(l)}^{2} \varphi_{i(l)}^{2 j(l)-\alpha(l)}=o\left(p_{0}^{2 r} \varphi^{-\alpha}\right)$. Hence, from the above, $b_{\alpha} D^{\alpha} f \in L_{2}\left(R^{k}\right)$ for any such $\alpha$.

Let $\theta^{*}(x) \in C_{0}^{\infty}\left(R^{1}\right)$, with $\theta^{*}(x)=1$ for $|x| \leq 1$, and $\theta^{*}(x)=0$ for $|x| \geq 2$. Let $\theta_{j}(x) \in C_{0}^{\infty}\left(R^{k}\right)$ denote $\prod_{i=1}^{k} \theta_{i}^{*}\left(x_{i} / j\right)$. It is clear, from the above, that as $j$ approaches infinity $L^{r}\left(\theta_{j} f\right)$ converges to $L f$ in $L_{2}\left(R^{k}\right)$ for all $r \leq n$. Hence $f \in$ domain $T_{0}\left(\prod_{i=1}^{n}\left(L+K_{i}\right)\right)$.

We now complete the proof as in Theorem 3.3. Let $\Pi$ denote $\prod_{i=1}^{n}\left(L+K_{i}\right)$. Suppose that the restriction of $\Pi$ to $C_{0}^{\infty}\left(R^{k}\right)$ is not essentially selfadjoint. Then there exists a $g \in C_{0}^{\infty}\left(R^{k}\right)$ such that $g \notin$ range $T_{0}(\Pi)$. Let $p_{0}^{2 n} g=h$, and find an $f$ in the domain of $G$ such that $G f=h$. Then $p_{0}^{2 n}(g-\Pi f)=0$; however, $f \in$ domain $T_{0}(\Pi)$, a contradiction. Hence the restriction of $\Pi$ to $C_{0}^{\infty}\left(R^{k}\right)$ is essentially selfadjoint. But for every $\varepsilon>0$, there exists a $K$ such that $\varepsilon \lambda^{n}+K \geq \lambda^{n-r}$ for all $\lambda \geq 1$ and $r \leq n$. Hence, by the spectral theorem, the restriction of $L^{n}-\Pi$ to the domain of $T_{0}(\Pi)$ is relatively bounded with respect to $T_{0}(\Pi)$, with relative bound zero. But therefore the restriction of $L^{n}$ to the domain of $T_{0}(\Pi)$ is closed. Hence this restriction contains $T_{0}\left(L^{n}\right)$. But similarly the restriction of $\Pi$ to the domain of $T_{0}\left(L^{n}\right)$ is a closed operator, which therefore contains $T_{0}(\Pi)$. Since the domain of $T_{0}\left(L^{n}\right)$ is contained in the domain of $T_{0}(\Pi)$, it follows that the two domains are equal. Hence $T_{0}\left(L^{n}\right)$ is selfadjoint, because it is a symmetric perturbation of a selfadjoint operator with relative bound zero. The theorem is proved.

THEOREM 6.5. Suppose that, for some n, the following property holds: for every $i \in 1, \ldots, k$ there exists a $j(i) \geq 1$ such that $p_{0}^{n} p_{i j(i)}$ is bounded away from zero. Suppose in addition that, for some $\varepsilon>0,\left(|x|^{2}+1\right)^{\varepsilon}=O\left(p_{0}^{2}\right)$. Then, if $\lambda_{j}$ is 
the spectrum of $T_{0}(L)$, listed in nondecreasing order according to multiplicity, there exists an $\varepsilon>0$ and $K>0$ such that $K+\lambda_{j} \geq j^{\varepsilon}$.

ProOF. By Lemma 6.3, with $q=0$, we may select $K_{i}$ such that

$$
\begin{aligned}
\left(\prod_{i=1}^{n}\left(L+K_{i}\right) f, f\right) & \geq \varepsilon_{1} S_{n} \\
& \geq \varepsilon_{1}\left(\left(\sum_{i=1}^{k}(-1)^{i} D_{i}^{j(i)} p_{i j(i)}^{2} p_{0}^{2 n} D_{i}^{j(i)}+p_{0}^{2 n}\right) f, f\right) \\
& \geq \varepsilon_{2}\left(\left(\sum_{i=1}^{k} D_{i}^{2 j(i)}+\left(|x|^{2}+1\right)^{n \varepsilon}\right) f, f\right) \\
& \geq \varepsilon_{3}\left(\left(\sum_{i=1}^{k} D_{i}^{2 j(i)}+\left(x_{i}^{2}+1\right)^{n \varepsilon}\right) f, f\right) \\
& \geq \varepsilon_{3}\left(\left(\sum_{i=1}^{k}(-1)^{i} D_{i}^{2}+\left(x_{i}^{2}+1\right)^{n \varepsilon}-K\right) f, f\right),
\end{aligned}
$$

for some $\varepsilon_{3}$ and $K>0$ and all $f \in C_{0}^{\infty}\left(R^{k}\right)$.

Since the restriction of $\prod_{i=1}^{n}\left(L+K_{i}\right)$ to $C_{0}^{\infty}\left(R^{k}\right)$ is essentially selfadjoint, this holds also for all $f$ in the domain of $T_{0}\left(\prod_{i=1}^{k}\left(L+K_{i}\right)\right)$. But, by separation of variables and Formula 7.17, p. 144, Titchmarsh [11], if $\beta_{n}$ is in the spectrum of $\sum_{i=1}^{k}(-1)^{i} D_{i}^{2}+\left(x_{i}^{2}+1\right)^{n \varepsilon}$, listed in increasing order, then $\beta_{j} \geq j^{\delta}$ for some $\delta>0$.

Note that $T_{0}\left(L^{n}\right)=T_{0}^{*}\left(L^{n}\right)=T_{1}\left(L^{n}\right)$, since the restriction of $L^{n}$ to $C_{0}^{\infty}\left(R^{k}\right)$ is essentially selfadjoint. But $T_{0}\left(L^{n}\right) \subseteq\left(T_{0}(L)\right)^{n} \subseteq T_{1}\left(L^{n}\right)$; hence $T_{0}\left(L^{n}\right)=$ $\left(T_{0}(L)\right)^{n}$. Thus the spectrum of $T_{0}\left(L^{n}\right)$, listed in nondecreasing order according to multiplicity, is $\lambda_{j}^{n}$, where $\lambda_{j}$ is as above. Let $\left\{\gamma_{j}\right\}$ be the spectrum of $T_{0}\left(\prod_{i=1}^{n}\left(L+K_{i}\right)\right)$, listed in nondecreasing order according to multiplicity. Note that since $\gamma_{j}$ and $\lambda_{j}^{n}$ are polynomials in $\lambda_{j}$ of the same degree, there exists a positive $K$ such that $\lambda_{j}^{n}+K \geq \gamma_{j}$. But, by inequality $6.1, \gamma_{j} \geq \varepsilon_{4} \beta_{j}-K$, for some positive $K$. Hence $\lambda_{j}^{n} \geq j^{\delta}$ when $j$ is large. The theorem is proved.

THEOREM 6.6. Suppose that the hypotheses of Theorem 6.5 hold, and that in addition $\alpha_{i}=o\left(p_{0}^{2}\right)$ for all $i \in 1, \ldots, k$. Then for any $\lambda$ such that $\operatorname{Re} \lambda>0$, the abstract Cauchy problem $d u(t) / d t=-\lambda L u, u(0)=f$, is well posed and $\rho$ contractive in $L_{2, \rho}\left(R^{k}\right)$, where $\omega^{-2}=\rho$, and where we use the notation of $\S 2$.

PROOF. This follows from Theorems 5.3 and 6.5.

\section{REFERENCES}

1. S. Agmon, Bounds on exponential decay of eigenfunctions of Schroedinger operators, Schroedinger Operators, Lecture Notes in Math., vol. 1159, Springer-Verlag, New York, 1984.

2. Lectures on exponential decay of solutions of second-order elliptic equations, Princeton Univ. Press, Princeton, N.J., 1982.

3. E. B. Davies and B. Simon, Ultracontractivity and the heat kernel for Schroedinger operators and Dirichlet laplacians' J. Funct. Anal. 59 (1984), 335-395.

4. W. D. Evans, On the essential self-adjointness of powers of Schroedinger operators, Proc. Roy. Soc. Edinburgh 79A (1977), 6177. 
5. J. Goldstein, Semigroups of linear operators and applications, Oxford Univ. Press, New York, 1985.

6. T. Kato, Remarks on self-adjointness and related problems for differential operators, Spectral Theory of Differential Operators (I. W. Knowles and R. T. Lewis, eds.), Math. Studies, no. 55, North-Holland, Amsterdam, 1981.

7. _ Perturbation theory for linear operators, Springer-Verlag, New York, 1966.

8. R. M. Kauffman, On essential self-adjointness of partial differential operators with positive coefficients, Proc. London Math. Soc. (3) 56 (1988).

9. E. Nelson, The free Markov field, J. Funct. Anal. 12 (1973), 211-227.

10. T. T. Read, On the essential self-adjointness of powers of Schroedinger operators, Proc. Roy. Soc. Edinburgh 97 A (1984), 233-246.

11. E. C. Titchmarsh, Eigenfunction expansions, Part 1, 2nd ed., Oxford, 1962.

Department of Mathematics, University of Alabama at Birmingham, BirMiNGHAM, Alabama 35294 